\title{
Poly ADP Ribose Polymerase Inhibitor Olaparib Targeting Microhomology End Joining in Retinoblastoma Protein Defective Cancer: Analysis of the Retinoblastoma Cell-Killing Effects by Olaparib after Inducing Double-Strand Breaks
}

\author{
Yuning Jiang ${ }^{1,2}$, Jason C. Yam ${ }^{1,3}$ and Wai Kit Chu ${ }^{1,3, *(D)}$ \\ 1 Department of Ophthalmology \& Visual Sciences, The Chinese University of Hong Kong, \\ Hong Kong 999077, China; xdk4xz@virginia.edu (Y.J.); yamcheuksing@cuhk.edu.hk (J.C.Y.) \\ 2 Department of Radiation Oncology, University of Virginia, Charlottesville, VA 22908, USA \\ 3 Hong Kong Hub of Paediatric Excellence, The Chinese University of Hong Kong, Hong Kong 999077, China \\ * Correspondence: waikit@cuhk.edu.hk
}

check for updates

Citation: Jiang, Y.; Yam, J.C.; Chu, W.K. Poly ADP Ribose Polymerase Inhibitor Olaparib Targeting Microhomology End Joining in Retinoblastoma Protein Defective Cancer: Analysis of the

Retinoblastoma Cell-Killing Effects by Olaparib after Inducing Double-Strand Breaks. Int. J. Mol. Sci. 2021, 22, 10687. https://doi.org/ 10.3390/ijms221910687

Academic Editors: Ireneusz Majsterek and Tomasz Poplawski

Received: 8 September 2021

Accepted: 28 September 2021

Published: 1 October 2021

Publisher's Note: MDPI stays neutral with regard to jurisdictional claims in published maps and institutional affiliations.

Copyright: (c) 2021 by the authors. Licensee MDPI, Basel, Switzerland. This article is an open access article distributed under the terms and conditions of the Creative Commons Attribution (CC BY) license (https:/ creativecommons.org/licenses/by/ $4.0 /)$
Abstract: Retinoblastoma is the most common intraocular cancer in childhood. Loss of function in both copies of the RB1 gene is the causal mutation of retinoblastoma. Current treatment for retinoblastoma includes the use of chemotherapeutic agents, such as the DNA damaging agent etoposide, which is a topoisomerase II poison that mainly generates DNA double-strand breaks (DSBs) and genome instability. Unfaithful repairing of DSBs could lead to secondary cancers and serious side effects. Previously, we found that RB knocked-down mammalian cells depend on a highly mutagenic pathway, the micro-homology mediated end joining (MMEJ) pathway, to repair DSBs. Poly ADP ribose polymerase 1 (PARP1) is a major protein in promoting the MMEJ pathway. In this study, we explored the effects of olaparib, a PARP inhibitor, in killing retinoblastoma cells. Retinoblastoma cell line Y79 and primary retinoblastoma cells expressed the cone-rod homeobox protein (CRX), a photoreceptor-specific marker. No detectable RB expression was found in these cells. The co-treatment of olaparib and etoposide led to enhanced cell death in both the Y79 cells and the primary retinoblastoma cells. Our results demonstrated the killing effects in retinoblastoma cells by PARP inhibitor olaparib after inducing DNA double-strand breaks. The use of olaparib in combination with etoposide could improve the cell-killing effects. Thus, lower dosages of etoposide can be used to treat retinoblastoma, which would potentially lead to a lower level of DSBs and a relatively more stable genome.

Keywords: PARP inhibitor; olaparib; etoposide; DNA double-strand break repair; retinoblastoma

\section{Introduction}

Retinoblastoma is the most common intraocular cancer in childhood. It accounts for approximately $3 \%$ of all childhood cancer [1]. The prevalence of retinoblastoma is approximately 1 in 15,000 live births. Eight thousand children are diagnosed with retinoblastoma each year worldwide [2]. Retinoblastoma patients usually show signs of leukocoria or strabismus. The median age of presentation is around 18 months. Retinoblastoma is a tumor occurring in the retina. It has been reported that retinoblastoma originates in the cone photoreceptor precursor cells [3]. Loss of both copies of the retinoblastoma (RB1) gene on chromosome 13q 14 is thought to be the most common cause of retinoblastoma [4]. In 1971, the "two-hit" mutation hypothesis was proposed to explain the cause of retinoblastoma [5], and the $R B 1$ gene was identified 16 years later [6]. $R B 1$ is the first tumor suppressor gene identified whose mutational inactivation would lead to the formation of a tumor [7]. Functional disruption of $R B 1$ can be generated by the somatic inactivation of both $R B 1$ alleles, or with a germline $R B 1$ mutation in one allele and a somatic inactivation of the second RB1 allele. This is described by the famous "two-hit hypothesis" to suggest the genetic 
mutation of both alleles of a single gene would lead to tumorigenesis [8]. In addition, inactivation of the RB1 gene could also happen in DNA sequence alteration, promoter methylation, and loss of heterozygosity [9]. Importantly, the risk of a second malignancy is very high in retinoblastoma patients. It was reported that during a 50 year period, around $46 \%$ retinoblastoma survivors developed a secondary cancer [10]. Current treatment for retinoblastoma aims to save the lives of patients, prevent the loss of eyes, and preserve useful vision. Depending on different clinical stages of retinoblastoma, the tumor may be confined to the retina (stage A-B), vitreous seeding may occur (stage $\mathrm{C}-\mathrm{D}$ ), or the condition may be unsalvageable (stage E) [11]. For intraocular retinoblastoma, several local treatments, such as laser, cryotherapy, radiation, and chemotherapy, are commonly used. At very late stages, enucleation to remove the eye is needed in order to save the life of the patient. For extraocular retinoblastoma, intensive chemotherapy, such as etoposide, is required [2]. Etoposide is a type II topoisomerase poison. Etoposide could generate DNA double-strand breaks (DSBs), which is considered the most toxic DNA damage due to the complete discontinuation of genetic materials. The severe side effects of chemotherapy, such as elevated risk of secondary cancer, including osteosarcoma and leukemia, is commonly observed in retinoblastoma patients [12]. In addition, diminished orbital growth, hearing loss, and enucleation would lead to a very low quality of life in patients.

The major cause of retinoblastoma was originally thought to be a consequence of mutations on both RB1 alleles [5]. Recent work has reported that the retention of a single functional RB1 allele is insufficient to maintain genome stability. Loss of one functional $R B 1$ allele results in DNA replication stress, accumulation of DSBs, mitotic errors, and dysregulation of p53 functions. The term "haploinsufficiency" is used to described the dosage effect in which the wild-type RB protein, generated from the single wild-type allele, is not sufficient to fulfil its functional requirements [13].

Recently, we reported that RB knocked-down U2OS cells could repair DSBs by employing a highly mutagenic micro-homology mediated end joining (MMEJ) pathway [14]. MMEJ is an error-prone DNA repair pathway. Several proteins, such as poly ADP ribose polymerase 1 (PARP1), polymerase theta (POLQ), and DNA ligase 3, are important regulators in MMEJ. In addition, our findings are consistent with previous studies of RB functions in promoting other DSB repair pathways, homologous recombination (HR), and canonical non-homologous end joining (C-NHEJ) $[15,16]$. Interestingly, previous research has sequenced flanking deletion breakpoints in the RB1 gene in retinoblastoma tumors and found 4 to $7 \mathrm{bp}$ direct repeat deletions. This suggests that the MMEJ pathway may be employed to repair DSBs in retinoblastoma cells [17]. Similar to our findings, some other cancers, such as breast cancer, are also defective in HR and could be treated with a PARP inhibitor [18]. Based on these observations, we evaluated the killing effects of the PARP inhibitor olaparib in retinoblastoma cells after inducing DSBs.

\section{Results}

\subsection{RB Deficient Cells Depend on MMEJ to Repair DSBs and Are Hypersensitive to} Etoposide Treatment

In our previous study, we observed that MMEJ efficiency was elevated in RB knockeddown cells. Therefore, we hypothesize that the PARP inhibitor olaparib could impede MMEJ in RB knocked-down cells. Our results demonstrated that RB knocked-down cells could significantly enhance MMEJ, while a one-hour treatment of the PARP inhibitor olaparib had no effect on MMEJ. Interestingly, olaparib treatment was able to suppress MMEJ significantly in RB knocked-down cells, suggesting that PARP is important for the elevated MMEJ in RB knocked-down cells (Figure 1). Next, we studied the DSB levels in U2OS cells treated with etoposide or olaparib, after knocking-down RB. Obvious RB loss could be observed in RB1 siRNA-treated U2OS cells. Equal expression levels of PARP1 were observed in RB knocked-down cells and control siRNA-treated cells, implying that RB loss would not affect the expression of PARP1 (Figure 2A). In both control siRNA-treated cells and RB1 siRNA-treated cells, significant elevation in the number of $\gamma \mathrm{H} 2 \mathrm{AX}$ foci, an 
indicator of DSB levels, could be observed after etoposide treatment, while the PARP1 inhibitor did not induce additional DSBs (Figure 2B).

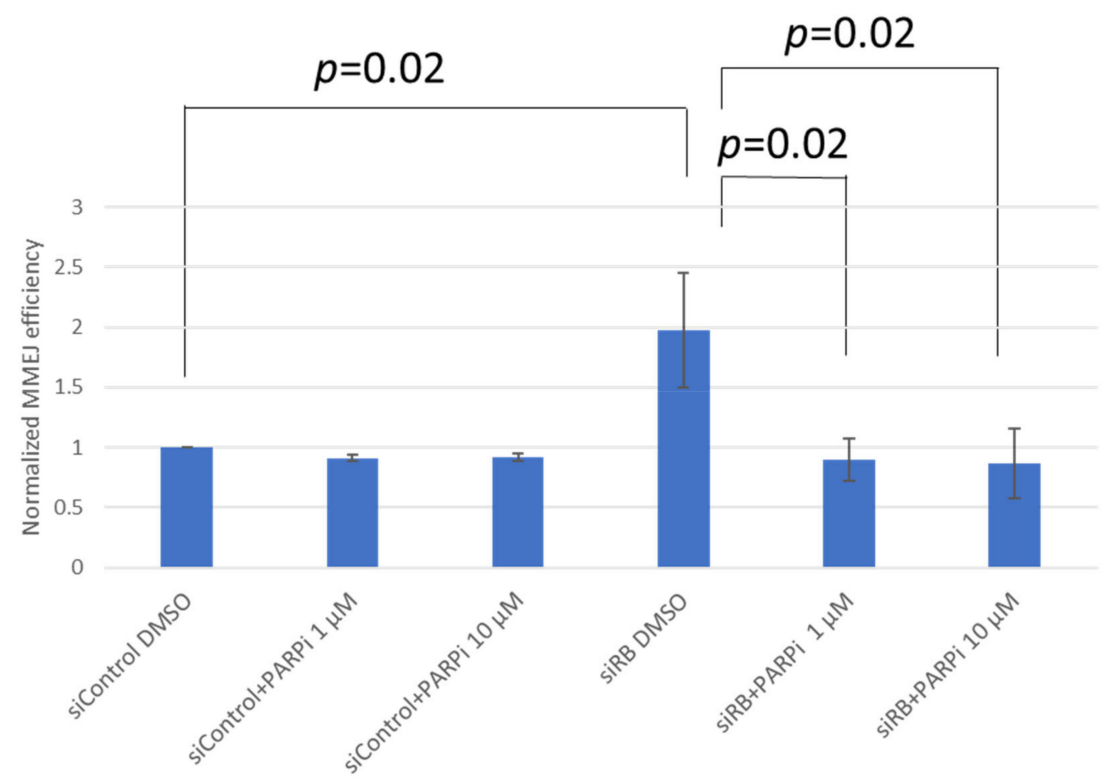

Figure 1. MMEJ efficiency was measured in non-targeting control siRNA (siControl) and RB siRNA (siRB)-transfected U2OS cells. Cells were also treated with DMSO, $1 \mu \mathrm{M}$ or $10 \mu \mathrm{M}$ olaparib (PARPi) for $1 \mathrm{~h}$. Experiments were repeated three times. Data are shown as mean \pm standard deviation and analyzed using two-tailed unpaired $t$-test.

A

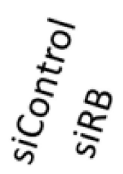

Relative normalized intensity 10.0752

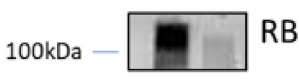

$50 \mathrm{kDa}-\longrightarrow$ Tubulin

B

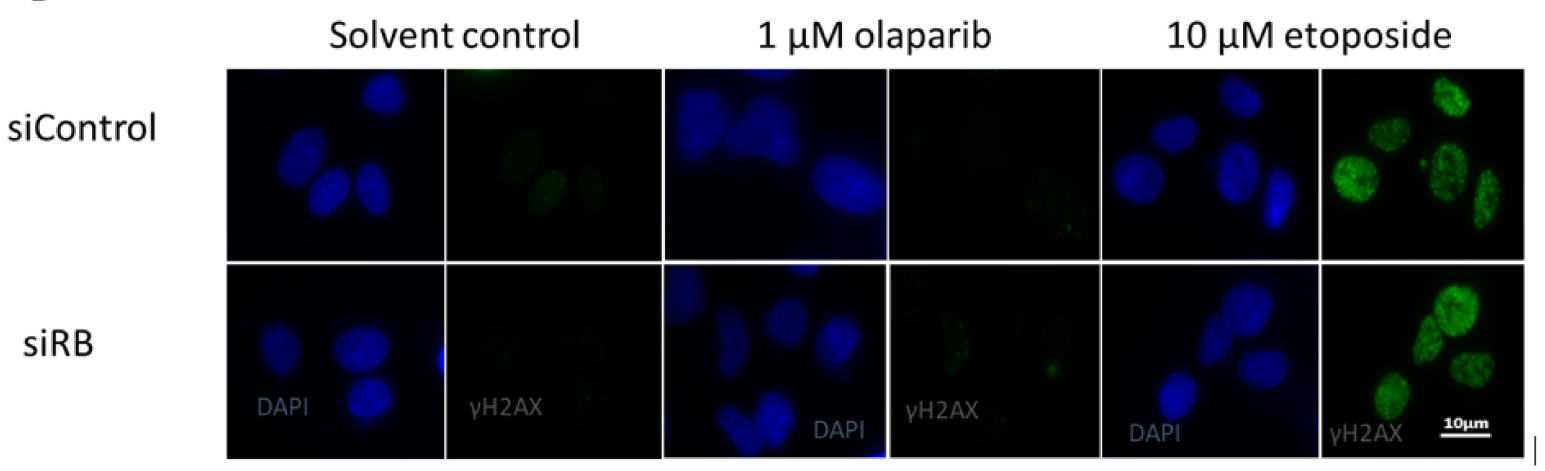

Figure 2. (A) RB was knocked-down efficiently three days after RB siRNA treatment, while PARP1 expression remained unchanged. GAPDH was used as the loading control. Western blot was repeated two times independently. Relative band intensities of RB and PARP1 are shown by normalizing with tubulin and GAPDH, respectively. (B) Immunofluorescence staining of $\gamma \mathrm{H} 2 \mathrm{AX}$ foci (green) in RB knocked-down cells (siRB) and control siRNA-treated cells (siControl) after treating with solvent control, $1 \mu \mathrm{M}$ olaparib or $10 \mu \mathrm{M}$ etoposide for one hour. Nuclei were stained with DAPI (blue). Immunofluorescence staining was repeated two times independently. Quantification of cells showing more than five $\gamma \mathrm{H} 2 \mathrm{AX}$ per cell is shown in Supplementary Figure S1. 
Unrepaired DSBs could lead to cell death. Cell survival was quantified by the MTT assay. RB knocked-down U2OS cells were hypersensitive to etoposide treatment. Significantly less cell survival was observed in RB knocked-down cells at $10 \mu \mathrm{M}$ and $25 \mu \mathrm{M}$ etoposide (Figure 3A). Significantly enhanced killing effects of RB knocked-down cells were also observed after treatment with $5 \mu \mathrm{M}$ and $10 \mu \mathrm{M}$ olaparib (Figure 3B). Based on our results, we noticed that no significantly enhanced killing could be observed in RB knocked-down cells treated with $5 \mu \mathrm{M}$ etoposide or $1 \mu \mathrm{M}$ olaparib (Figure 3A,B). Therefore, we explored if the co-treatment of etoposide and olaparib could improve the insignificant killing effects at $5 \mu \mathrm{M}$ etoposide and $1 \mu \mathrm{M}$ olaparib. In our first experiment, we co-treated various concentrations of etoposide with $1 \mu \mathrm{M}$ olaparib and observed significantly less cell survival in RB knocked-down cells (Figure 3C). Then, we co-treated various concentrations of olaparib with $5 \mu \mathrm{M}$ etoposide. Significantly less cell survival could also be detected under these conditions in RB knocked-down cells (Figure 3D). Our results demonstrated that the co-treatment of etoposide and olaparib could improve the cell killing effects of single-drug treatments at dosages that had no significant effects on cell survival.

A

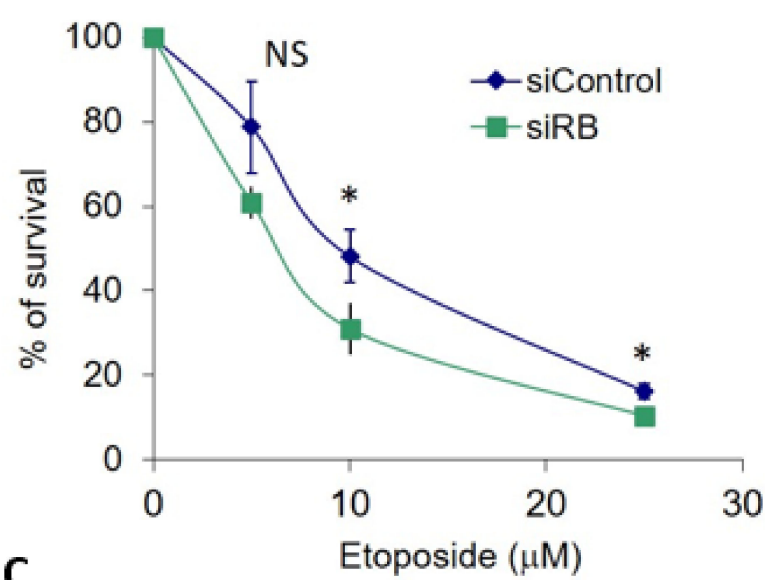

C

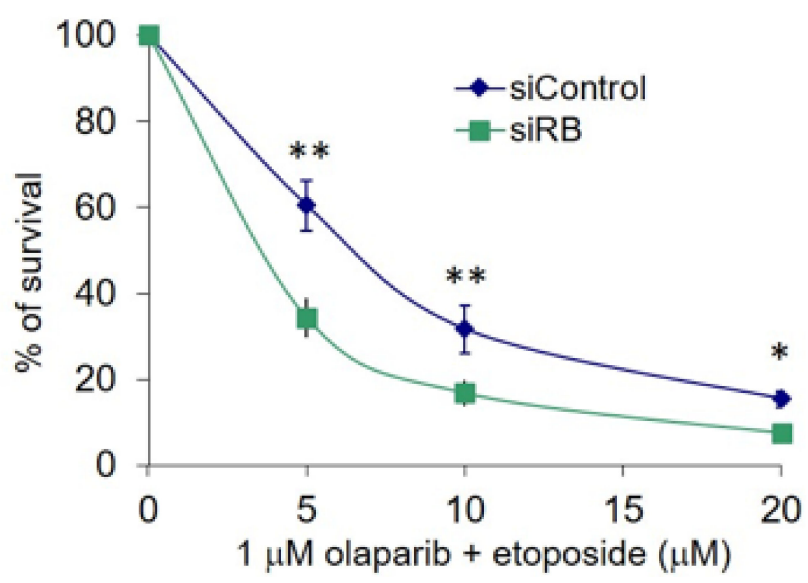

B

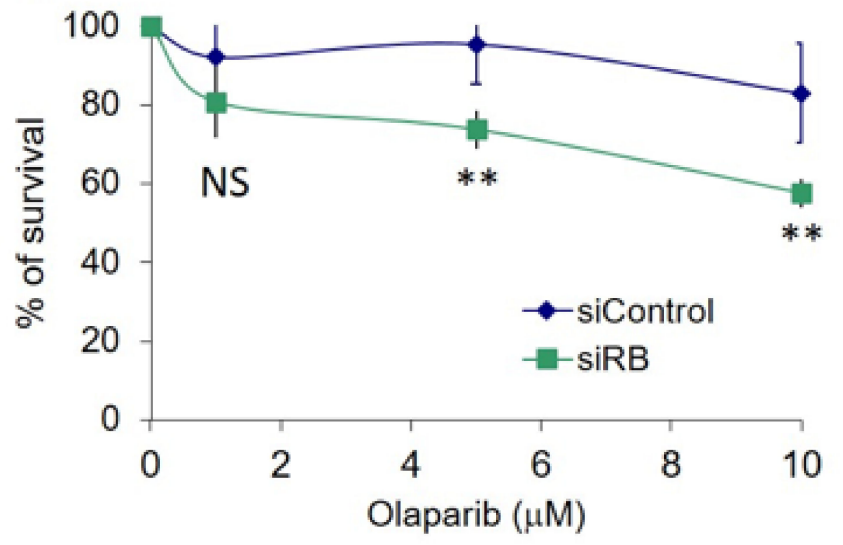

D

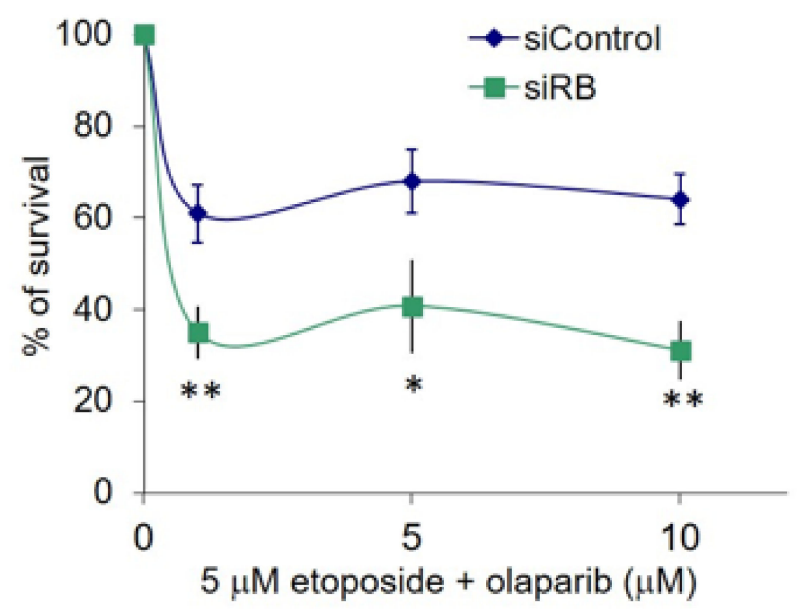

Figure 3. (A) MTT assay in RB knocked-down (siRB) and control siRNA (siControl)-treated U2OS cells showed cell survival after treating various dosages of etoposide for three days. (B) MTT assay in RB knocked-down and control siRNA-treated U2OS cells showed cell survival after treatment with various dosages of olaparib for three days. (C) MTT assay in RB knocked-down and control siRNA-treated U2OS cells showed cell survival after co-treatment with $1 \mu \mathrm{M}$ olaparib with various dosages of etoposide for three days. (D) MTT assay in RB knocked-down and control siRNA-treated U2OS cells showed cell survival after co-treatment with $5 \mu \mathrm{M}$ etoposide with various dosages of olaparib for three days. Experiments were repeated three times. Data are shown as mean \pm standard deviation and analyzed using two-tailed unpaired $t$-test: * represents $p<0.05,{ }^{* *}$ represents $p<0.01$, NS represents no significance. 


\title{
2.2. RB Expression in Primary Retinoblastoma Cells
}

To further evaluate the treatment effects in retinoblastoma, the effects of the cotreatment of olaparib and etoposide were also studied in primary retinoblastoma cells. Primary retinoblastoma cells were isolated from an enucleated tumor from a female unilateral retinoblastoma patient diagnosed at 1 year of age. The tumor was localized in the right eye in connection with the posterior side of the lens, the ciliary body, and the iridocorneal angle. Genetic analysis by the multiplex ligation-dependent robe amplification (MLPA), DNA sequencing, and allele-specific polymerase chain reaction found that both $R B 1$ alleles possessed a mutation of c.470_473delins450_461 ( $p$.Val157AspfsTer3). This mutation led to a deletion between position 470 and 473 and an insertion from 450 to 461 in the coding DNA sequence, resulting in an amino acid substitution valine to aspartic acid at position 157 and a stop codon termination at position 159. No RB1 mutation was identified in the blood sample. In the primary retinoblastoma cells isolated from this patient, as well as the Y79 cells, a commercially available retinoblastoma cell line, the photoreceptor specific protein CRX expression was detectable (Figure 4A,B), confirming that these cells were developed from photoreceptors. No RB expression could be detected in the primary retinoblastoma cells and the Y79 cells (Figure 4C,D).

A

\begin{abstract}
RB primary + secondary antibodies

Secondary antibody only
\end{abstract}

C

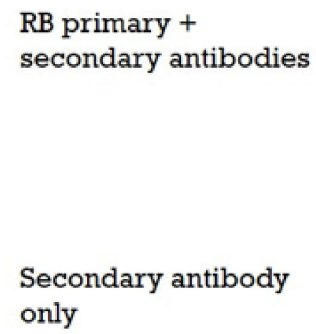

Primary retinoblastoma cells

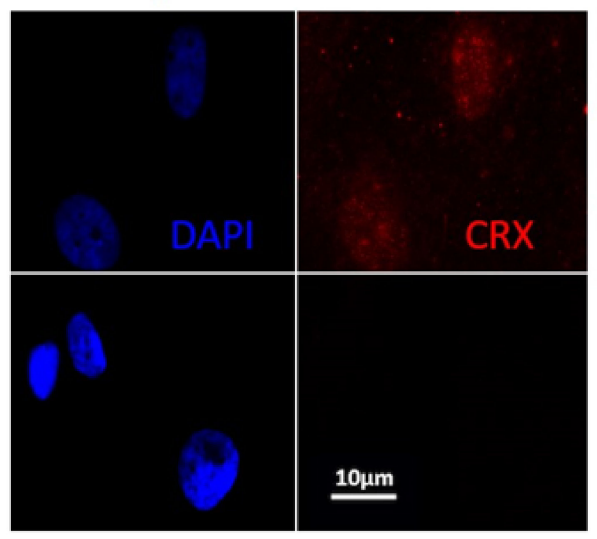

Primary retinoblastoma cells

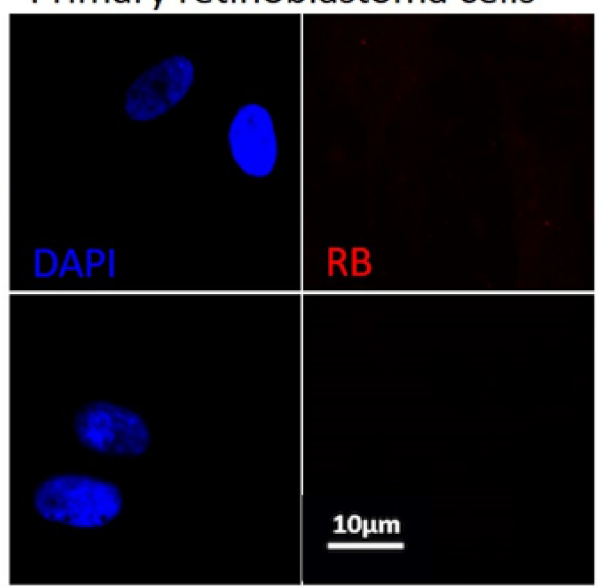

B

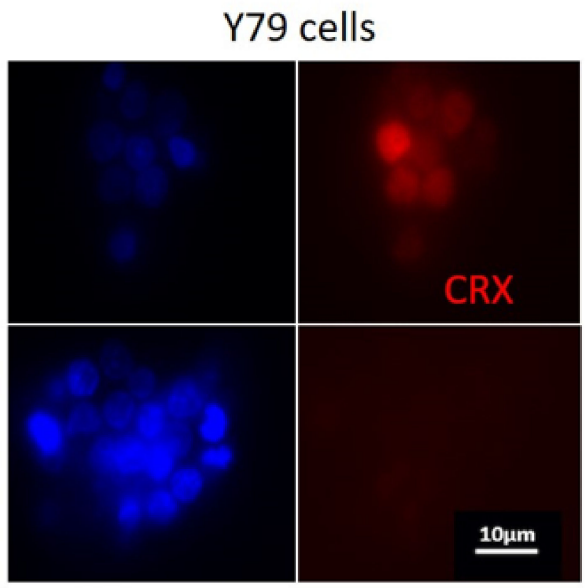

D

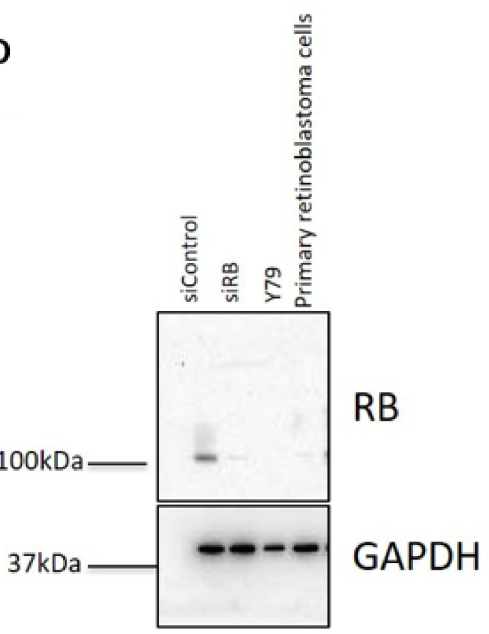

Figure 4. (A) Immunofluorescence staining detected CRX (red) in primary retinoblastoma cells. (B) CRX (red) was also detected in Y79 cells. (C) Immunofluorescence staining showed loss of RB signal (red) in primary retinoblastoma cells. Nuclei were stained with DAPI (blue). (D) RB was only detected in control siRNA (siControl)-treated U2OS cells. No RB could be detected in RB knocked-down (siRB) U2OS cells, Y79 cells or primary retinoblastoma cells. GAPDH was used as the loading control. 


\subsection{Olaparib and Etoposide Co-Treatment in Primary Retinoblastoma Cells}

Our results from the RB knocked-down U2OS cells demonstrated that the co-treatment of etoposide and olaparib could improve the cell killing effects. Therefore, we investigated the cell killing effects in the primary retinoblastoma cells co-treated with different dosages of etoposide in the presence of $1 \mu \mathrm{M}$ olaparib. Enhanced killing effects could be observed in cells treated with $1 \mu \mathrm{M}$ olaparib plus $10 \mu \mathrm{M}$ or $25 \mu \mathrm{M}$ etoposide (Figure $5 \mathrm{~A}, \mathrm{~B}$ ).

A

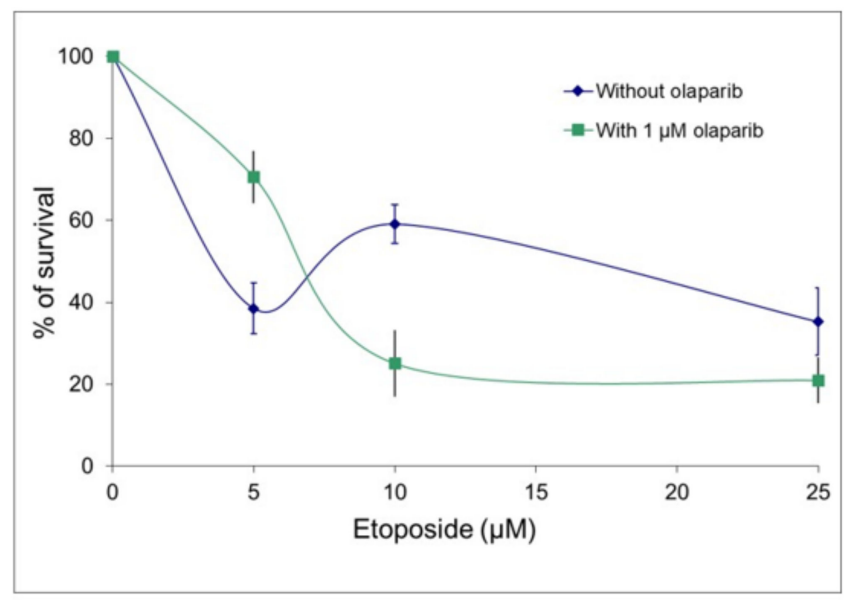

B

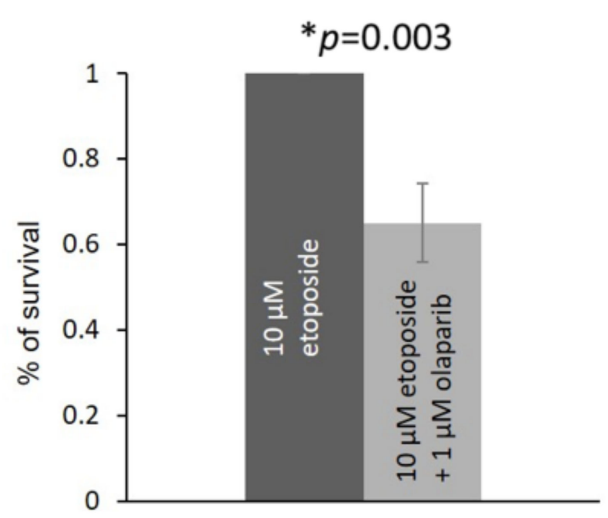

Figure 5. (A) MTT assay in primary retinoblastoma cells showed cell survival after co-treatment with $1 \mu \mathrm{M}$ olaparib with various dosages of etoposide for three days. Experiments were repeated three times. Data are shown as mean \pm standard deviation. (B) MTT assay in primary retinoblastoma cells showed cell survival after co-treatment with $1 \mu \mathrm{M}$ olaparib and $10 \mu \mathrm{M}$ etoposide for three days. Experiments were repeated three times. Data are shown as mean \pm standard deviation. and analyzed using two-tailed unpaired $t$-test. ${ }^{*}$ represents statistical significance $p=0.003$.

We also studied the effects on cell proliferation in another retinoblastoma cell line, Y79. As Y79 cells are in suspension in cell culture, EdU DNA synthesis assay was used to studied cell proliferation. Comparing Y79 cells treated with $10 \mu \mathrm{M}$ etoposide, Y79 cells co-treated with $1 \mu \mathrm{M}$ olaparib plus $10 \mu \mathrm{M}$ etoposide showed significantly less proliferation (Figure 6). 


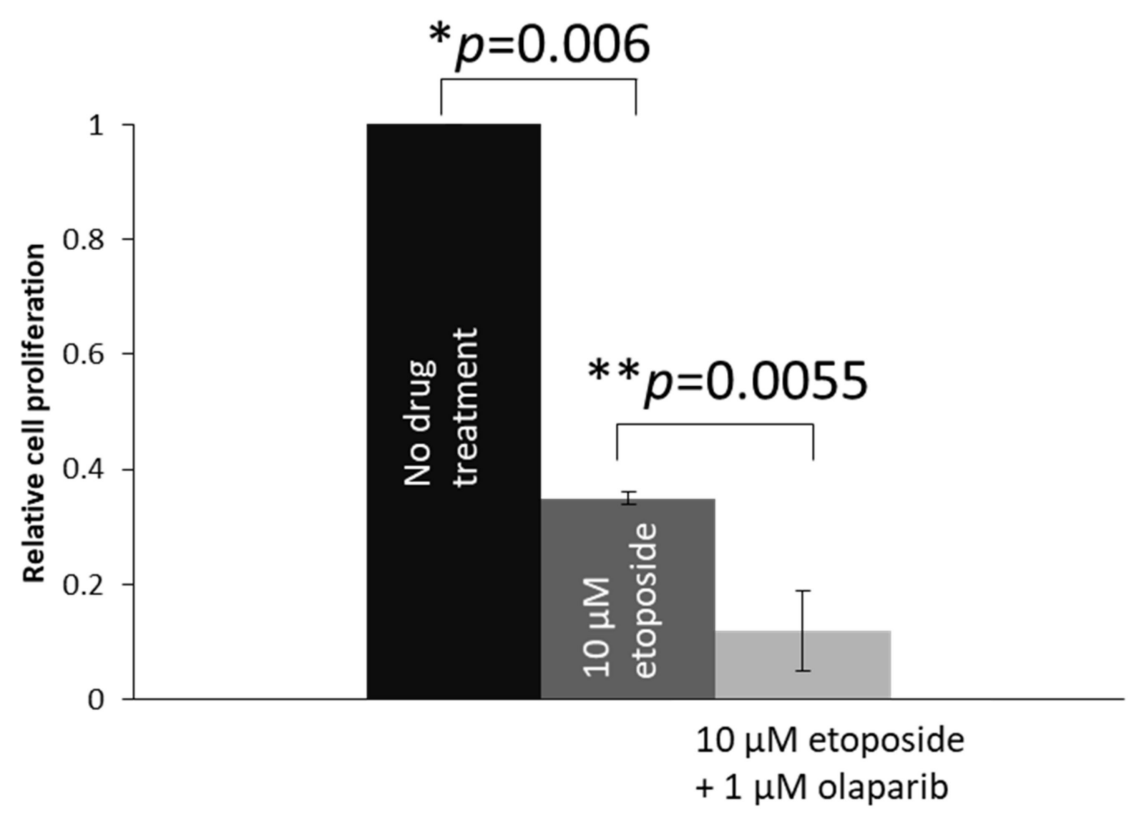

Figure 6. Cell proliferation analysis by using the EdU synthesis assay. Proliferation of the $1 \mu \mathrm{M}$ Olaparib- and $10 \mu \mathrm{M}$ etoposide-co-treated cells was significantly lower than the $10 \mu \mathrm{M}$ etoposidetreated cells. Experiments were repeated three times. Data are shown as mean \pm standard deviation and analyzed using two-tailed unpaired $\mathrm{t}$ test. ${ }^{*}$ and ${ }^{* *}$ represent statistical significance of $p=0.006$ and $p=0.0055$, respectively.

\section{Discussion}

We have previously reported that RB knocked-down cells are highly dependent on MMEJ to repair DSBs. In this study, we showed that the PARP inhibitor olaparib significantly inhibited MMEJ in RB knocked-down cells by quantifying MMEJ efficiency after PARP inhibitor treatment. Importantly, in these RB knocked-down cells, we showed that the co-treatment of etoposide and olaparib could improve the cell killing effects of single-drug treatments at dosages that had no significant effects on cell survival. We further showed in the primary retinoblastoma cells that the co-treatment of etoposide with higher dosages $(>10 \mu \mathrm{M})$ of etoposide was able to enhance cell killing, compared to the etoposide single treatment. However, a lower dose of etoposide $(5 \mu \mathrm{M})$ did not show enhanced cell killing effects in combination with $1 \mu \mathrm{M}$ olaparib in the primary retinoblastoma cells, while the enhanced killing effects were still observed in RB knocked-down U2OS cells. Currently, it is unclear why there were different killing effects between these two cellular systems. Possible explanations may be related to the slower cell proliferation of the primary retinoblastoma cells than $\mathrm{U} 2 \mathrm{OS}$ cells, which doubled around $72 \mathrm{~h}$ and $24 \mathrm{~h}$, respectively. A slower cell proliferation may contribute to less sensitivity to the co-treatment of olaparib with a low dose of etoposide. Future studies are needed to study the molecular mechanism under the co-treatment of olaparib with etoposide.

Etoposide is type II topoisomerase poison that has been widely used to treat cancer patients [19]. A PARP inhibitor is currently used to induce synthetic lethality in BRCAdeficient cancer [20]. BRCA is an important protein to promote HR. BRCA1 and 2-deficient cells also showed hypersensitivities to DSBs induced by etoposide [21]. Previous studies also showed RB functions to promote HR and C-NHEJ [15,16]. In addition, we demonstrated that RB-deficient cells are defective in HR and C-NHEJ and highly dependent on MMEJ to repair DSBs [14]. Specifically blocking MMEJ would be effective in killing retinoblastoma cells without damaging non-cancerous cells. On the other hand, PARP1 also functions to promote base excision repair (BER), which mainly repairs single strand breaks (SSBs) and protects cells against the generation of excessive SSBs during BER [22]. Besides $R B 1$, there is another gene, $M Y C N$, which is the most commonly amplified gene found in retinoblastoma [23]. It has been reported that a PARP inhibitor could enhance DNA 
replication stress and cause mitotic catastrophe in $M Y C N$ dependent neuroblastoma [24]. Whether the use of a PARP inhibitor is effective in treating MYCN mutated retinoblastoma will require further investigation. Genetically, retinoblastoma can occur in two forms: the heritable form more often results in bilateral retinoblastoma, while the non-heritable form is usually unilateral retinoblastoma. Approximately $45 \%$ of children with retinoblastoma are in the heritable form [25]. For patients possessing heritable retinoblastoma, at least one copy of the tumor initiating RB1 mutations could also be found in other non-cancerous cells, which could lead to lower RB protein levels and higher MMEJ efficiency in these cells. If heritable retinoblastoma patients are treated systematically with a PARP inhibitor, the MMEJ pathway might also be inhibited in the non-cancerous cells, which would lead to potentially serious side effects, including synthetic lethality in these non-cancerous cells. Local PARP inhibitor treatment could be more suitable for the heritable retinoblastoma patients.

The high prevalence of secondary cancer is another serious problem for retinoblastoma survivors following recovery [26]. Whether this high secondary cancer prevalence is related to chemotherapy is not very well understood. MMEJ is a highly mutagenic pathway to repair DSBs induced by DNA-damaging drugs, such as etoposide, which can also lead to genome instability. An unstable genome would have a higher chance of accumulating further mutations that would lead to secondary cancers in retinoblastoma patients. The use of olaparib in combination with etoposide could improve the cell killing effects. Thus, lower dosages of etoposide can be used to treat retinoblastoma, which would potentially lead to lower level of DSBs and a relatively more stable genome. Further long-term animal studies are needed to evaluate the prevalence of secondary cancer after receiving the combination low-dose treatment of olaparib and etoposide.

\section{Methods}

\subsection{Cell Culture and siRNA Transfection}

Human U2OS cells were obtained from ATCC and maintained in low-glucose DMEM medium (Gibco, Amarillo, TX, USA) supplemented with 10\% fetal bovine serum and $1 \%$ antibiotics ( $100 \mathrm{U} / \mathrm{mL}$ penicillin and $100 \mathrm{\mu g} / \mathrm{mL}$ streptomycin, Gibco, Amarillo, TX, USA). Cells were transfected with $100 \mathrm{nM}$ control siRNA (siControl): 5'-UGGUUUACAUGUCGACUAA-3' (Dharmacon, Lafayette, CO, USA) and RB1 siRNA (siRB): 5'-GAAAUGACUUCUACUCGAA$3^{\prime}$ (Sigma-Aldrich, Burlington, MA, USA). siRNA was transfected to cells with DharmaFECT1 (Dharmacon, Lafayette, CO, USA) for $72 \mathrm{~h}$. Human Y79 cells were obtained from ATCC, and primary retinoblastoma cells were isolated from the retinoblastoma tumors. The retinoblastoma tumor was cut out from the eyeball and dissociated with gentle pipetting. Primary retinoblastoma cells within the first five passages were used in this study. Both retinoblastoma cells were cultured in RPMI 1640 medium (Gibco, Amarillo, TX, USA) supplemented with 20\% fetal bovine serum and $1 \%$ antibiotics $(100 \mathrm{U} / \mathrm{mL}$ penicillin and $100 \mu \mathrm{g} / \mathrm{mL}$ streptomycin, Gibco, Amarillo, TX, USA). Both U2OS and retinoblastoma cells were maintained at $37^{\circ} \mathrm{C}$ in an incubator with $5 \% \mathrm{CO}_{2}$ and $95 \%$ air.

\subsection{MMEJ Reporter Assay}

In the reporter assay, $2.5 \times 10^{5}$ cells were transfected with siControl and siRB for 3 days. Cells were then treated with DMSO, $1 \mu \mathrm{M}$ or $10 \mu \mathrm{M}$ olaparib for $1 \mathrm{~h}$ in both siControl- and siRB-treated cells. Cells were then transfected with the EJ2GFP (Addgene, Watertown, MA, USA) to quantify MMEJ efficiency. Furthermore, $1 \mu \mathrm{g}$ EJ2GFP plasmids was transfected along with $1 \mu \mathrm{g}$ pCBASceI (Addgene, Watertown, MA, USA) to cells with Fugene6 (Promega, Madison, WI, USA). The ratio of Fugene6 to the transfected plasmid was 3:1. In another round of transfection, the same numbers of cells transfected with $2 \mu \mathrm{g}$ red fluorescent protein (RFP) expression plasmid pCAG-DsRed (Addgene, Watertown, MA, USA) were used to normalize the transfection efficiency [27]. Cells were then incubated for $72 \mathrm{~h}$ after transfection and collected for flow cytometry analysis. 


\subsection{MTT Cell Proliferation Assay}

In the cell proliferation assay, $1.5 \times 10^{6}$ U2OS cells were seeded in $10 \mathrm{~cm}$ dishes and treated with siControl or siRB for $48 \mathrm{~h}$. Cells were then collected. Furthermore, $1 \times 10^{5}$ cells were seeded into a 24 -well plate. After $24 \mathrm{~h}$, cells were treated with different dosages of etoposide and olaparib for three days. Cells were then incubated with $5 \mathrm{mg} / \mathrm{mL}$ MTT solution (Life technologies, Waltham, MA, USA) in $500 \mu \mathrm{L}$ DMEM medium at room temperature for $3 \mathrm{~h}$. MTT solution was then removed, and $300 \mu \mathrm{L}$ isopropanol was added into each well. An amount of $200 \mu \mathrm{L}$ of the mixture was transferred to a 96-well plate. Fluorescence signals were measured by a plate reader at $570 \mathrm{~nm}$ wavelength. To study the cell proliferation of primary retinoblastoma cells, cells were treated with etoposide and olaparib for three days, and cell proliferation was measured by MTT following the same protocol.

\subsection{Immunofluorescence}

Primary retinoblastoma cells and U2OS cells were seeded on glass coverslips. After $24 \mathrm{~h}$, cells were left untreated, or treated with olaparib and etoposide for one hour. Cells were then fixed in $3.7 \%$ para-formaldehyde in phosphate buffered saline (PBS) for $10 \mathrm{~min}$, washed in PBS and stored at $4{ }^{\circ} \mathrm{C}$. After permeabilization with $0.1 \%$ TritonX-100 in PBS, cells were washed in freshly made blocking buffer $(0.5 \mathrm{~g}$ BSA and $0.15 \mathrm{~g}$ glycine in $50 \mathrm{~mL}$ PBS). Cells were then incubated with primary antibodies against $\gamma \mathrm{H} 2 \mathrm{AX}(1: 200$, ab26350, Abcam, Cambridge, UK), CRX (1:200, sc-377138, Santa Cruz Biotechnology, Dallas, TX, USA) or RB antibody (1:200, ab181616, Abcam, Cambridge, UK). Cells were then incubated with donkey anti-mouse IgG AF488 (1:800, A21202, Invitrogen, Waltham, MA, USA) or donkey anti-rabbit IgG AF488 (1:800, A21206, Invitrogen, Waltham, MA, USA). The nuclei were mounted with DAPI (Vectorlabs, Burlingame, CA, USA) before coverslips were sealed to glass slides. Y79 cells were stained with the same procedures. $\gamma \mathrm{H} 2 \mathrm{AX}, \mathrm{CRX}$ and RB signals were visualized with a fluorescence microscope (Nikon, Tokyo, Japan), and images were analyzed by an imaging software (SPOT Imaging Solutions, Sterling Heights, MI, USA, version 5.2).

\subsection{Western Blotting}

Cells were lysed in Laemmli sample buffer. After protein quantification by the BCA protein assay, protein samples were boiled with BB buffer (0.5M DTT, $0.25 \%$ bromophenol blue $(w / v))$ at $95^{\circ} \mathrm{C}$ for $5 \mathrm{~min}$. The denatured proteins were separated in sodium dodecyl sulfate-polyacrylamide gel and then transferred to nitrocellular membranes. Membranes were probed with primary antibodies against RB (1:500, ab181616, Abcam, Cambridge, UK) and PARP1 (1:500, MA5-15031, ThermoFisher, Waltham, MA, USA), followed by incubation with appropriate secondary antibodies coupled with horseradish peroxidase. Membranes were then incubated with the enhanced chemiluminescence substrate, and images were captured. Band intensities were quantified by using ImageJ (National Institutes of Health, Bethesda, MD, USA, version 1.52a).

\subsection{Click-It Plus Edu Flow Cytometry Assay to Detect Cell Proliferation}

In the cell proliferation assay, $4 \times 10^{5}$ Y79 cells were seeded in a 6-well plate. After treatment with $10 \mu \mathrm{M}$ etoposide or co-treatment with $10 \mu \mathrm{M}$ etoposide and $1 \mu \mathrm{M}$ olaparib for 3 days, cells were treated with $10 \mu \mathrm{M}$ EdU for $2 \mathrm{~h}$. Cells were then harvested and fixed by $100 \%$ ethanol. Cells were stained with the Click-iT Plus reaction cocktail (Life technologies, Carlsbad, CA, USA) following the manufacturer's protocol and analyzed with flow cytometry. Relative cell proliferation was calculated by dividing the percentage of EdU positive cells in the drug-treated sample by the percentage of EdU-positive cells in the untreated sample. 
Supplementary Materials: The following are available online at https:/ / www.mdpi.com/article/10 $.3390 /$ ijms221910687/s1, Figure S1: Quantification of cells showing more than five $\gamma \mathrm{H} 2 \mathrm{AX}$ per cell.

Author Contributions: Y.J. and W.K.C. designed the study. Y.J. performed the experiments. Y.J., J.C.Y., and W.K.C. analyzed the results. Y.J. and W.K.C. wrote the manuscript. All authors have read and agreed to the published version of the manuscript.

Funding: This research and the APC were supported by The Chinese University of Hong Kong Direct Grant (4054360, to W.K.C.), the Health and Medical Research Fund, the Food and Health Bureau, The Government of the Hong Kong Special Administrative Region (07180306 and PR-HKCH-8 to J.C.Y., 06170896 to J.C.Y. and W.K.C.).

Institutional Review Board Statement: This study adhered to the tenets of the Declaration of Helsinki and was approved by the institutional review board of Hospital Authority, Hong Kong (reference number KC/KE-18-0097/ER-1).

Informed Consent Statement: Informed consent was obtained from all subjects involved in the study.

Conflicts of Interest: The authors declare no conflict of interest.

\section{References}

1. Rodriguez-Galindo, C.; Wilson, M.W.; Haik, B.G.; Lipson, M.J.; Cain, A.; E Merchant, T.; Kaste, S.; Pratt, C.B. Treatment of metastatic retinoblastoma. Ophthalmol. 2003, 110, 1237-1240. [CrossRef]

2. Lohmann, D. Retinoblastoma. Adv. Exp. Med. Biol. 2010, 685, 220-227.

3. Xu, X.L.; Fang, Y.; Lee, T.C.; Forrest, D.; Gregory-Evans, C.; Almeida, D.; Liu, A.; Jhanwar, S.C.; Abramson, D.H.; Cobrinik, D. Retinoblastoma Has Properties of a Cone Precursor Tumor and Depends Upon Cone-Specific MDM2 Signaling. Cell 2009, 137, 1018-1031. [CrossRef]

4. Friend, S.H.; Bernards, R.; Rogelj, S.; Weinberg, R.A.; Rapaport, J.M.; Albert, D.M.; Dryja, T.P. A human DNA segment with properties of the gene that predisposes to retinoblastoma and osteosarcoma. Nat. Cell Biol. 1986, 323, 643-646. [CrossRef] [PubMed]

5. Knudson, A.G. Mutation and Cancer: Statistical Study of Retinoblastoma. Proc. Natl. Acad. Sci. USA 1971, 68, 820-823. [CrossRef] [PubMed]

6. Fung, Y.; Murphree, A.; T'Ang, A.; Qian, J.; Hinrichs, S.; Benedict, W. Structural evidence for the authenticity of the human retinoblastoma gene. Science 1987, 236, 1657-1661. [CrossRef]

7. Berry, J.L.; Polski, A.; Cavenee, W.K.; Dryja, T.P.; Murphree, A.L.; Gallie, B.L. The RB1 Story: Characterization and Cloning of the First Tumor Suppressor Gene. Genes 2019, 10, 879. [CrossRef] [PubMed]

8. Comings, D.E. A General Theory of Carcinogenesis. Proc. Natl. Acad. Sci. USA 1973, 70, 3324-3328. [CrossRef]

9. Choy, K.W.; Pang, C.P.; Yu, C.B.; Wong, H.L.; Ng, J.S.; Fan, D.S.; Lo, K.W.; Chai, J.T.; Wang, J.; Fu, W.; et al. Loss of heterozygosity and mutations are the major mechanisms of RB1 gene inactivation in Chinese with sporadic retinoblastoma. Hum. Mutat. 2002, 20, 408. [CrossRef]

10. Mohney, B.G.; Robertson, D.M.; Schomberg, P.J.; O Hodge, D. Second nonocular tumors in survivors of heritable retinoblastoma and prior radiation therapy. Am. J. Ophthalmol. 1998, 126, 269-277. [CrossRef]

11. Shields, C.L.; Mashayekhi, A.; Au, A.K.; Czyz, C.; Leahey, A.; Meadows, A.T.; Shields, J.A. The International Classification of Retinoblastoma Predicts Chemoreduction Success. Ophthalmology 2006, 113, 2276-2280. [CrossRef] [PubMed]

12. Temming, P.; Arendt, M.; Viehmann, A.; Eisele, L.; Le Guin, C.H.D.; Schündeln, M.M.; Biewald, E.; Astrahantseff, K.; Wieland, R.; Bornfeld, N.; et al. Incidence of second cancers after radiotherapy and systemic chemotherapy in heritable retinoblastoma survivors: A report from the German reference center. Pediatr. Blood Cancer 2017, 64, 71-80. [CrossRef] [PubMed]

13. Ishak, C.; A Dick, F. Conditional haploinsufficiency of the retinoblastoma tumor suppressor gene. Mol. Cell. Oncol. 2014, 2, e968069. [CrossRef] [PubMed]

14. Jiang, Y.; Yam, J.C.; Tham, C.C.; Pang, C.P.; Chu, W.K. RB Regulates DNA Double Strand Break Repair Pathway Choice by Mediating CtIP Dependent End Resection. Int. J. Mol. Sci. 2020, 21, 9176. [CrossRef]

15. Cook, R.; Zoumpoulidou, G.; Luczynski, M.T.; Rieger, S.; Moquet, J.; Spanswick, V.J.; Hartley, J.A.; Rothkamm, K.; Huang, P.H.; Mittnacht, S. Direct Involvement of Retinoblastoma Family Proteins in DNA Repair by Non-homologous End-Joining. Cell Rep. 2015, 10, 2006-2018. [CrossRef]

16. Velez-Cruz, R.; Manickavinayaham, S.; Biswas, A.K.; Clary, R.W.; Premkumar, T.; Cole, F.; Johnson, D.G. RB localizes to DNA double-strand breaks and promotes DNA end resection and homologous recombination through the recruitment of BRG1. Genes Dev. 2016, 30, 2500-2512. [CrossRef]

17. Canning, S.; Dryja, T.P. Short, direct repeats at the breakpoints of deletions of the retinoblastoma gene. Proc. Natl. Acad. Sci. USA 1989, 86, 5044-5048. [CrossRef] 
18. Chopra, N.; Tovey, H.; Pearson, A.; Cutts, R.; Toms, C.; Proszek, P.; Hubank, M.; Dowsett, M.; Dodson, A.; Daley, F.; et al. Homologous recombination DNA repair deficiency and PARP inhibition activity in primary triple negative breast cancer. Nat. Commun. 2020, 11, 2662. [CrossRef]

19. Delgado, J.L.; Hsieh, C.-M.; Chan, N.-L.; Hiasa, H. Topoisomerases as anticancer targets. Biochem. J. 2018, 475, 373-398. [CrossRef]

20. Ashworth, A. A Synthetic Lethal Therapeutic Approach: Poly (ADP) Ribose Polymerase Inhibitors for the Treatment of Cancers Deficient in DNA Double-Strand Break Repair. J. Clin. Oncol. 2008, 26, 3785-3790. [CrossRef]

21. Treszezamsky, A.D.; Kachnic, L.A.; Feng, Z.; Zhang, J.; Tokadjian, C.; Powell, S.N. BRCA1-and BRCA2-Deficient Cells Are Sensitive to Etoposide-Induced DNA Double-Strand Breaks via Topoisomerase II. Cancer Res. 2007, 67, 7078-7081. [CrossRef] [PubMed]

22. Krokan, H.E.; Bjoras, M. Base excision repair. Cold Spring Harb. Perspect. Biol. 2013, 5, a012583. [CrossRef] [PubMed]

23. McEvoy, J.; Nagahawatte, P.; Finkelstein, D.; Richards-Yutz, J.; Valentine, M.; Ma, J.; Mullighan, C.; Song, G.; Chen, X.; Wilson, M.; et al. RB1 gene inactivation by chromothripsis in human retinoblastoma. Oncotarget 2014, 5, 438-450. [CrossRef] [PubMed]

24. Colicchia, V.; Petroni, M.; Guarguaglini, G.; Sardina, F.; Sahún-Roncero, M.; Carbonari, M.; Ricci, B.; Heil, C.; Capalbo, C.; Belardinilli, F.; et al. PARP inhibitors enhance replication stress and cause mitotic catastrophe in MYCN-dependent neuroblastoma. Oncogene 2017, 36, 4682-4691. [CrossRef]

25. Yun, J.; Li, Y.; Xu, C.-T.; Pan, B.-R. Epidemiology and Rb1 gene of retinoblastoma. Int. J. Ophthalmol. 2011, 4, 103-109. [CrossRef]

26. Kleinerman, R.A.; Tucker, M.A.; Tarone, R.E.; Abramson, D.H.; Seddon, J.M.; Stovall, M.; Li, F.P.; Jr, J.F.F. Risk of New Cancers After Radiotherapy in Long-Term Survivors of Retinoblastoma: An Extended Follow-Up. J. Clin. Oncol. 2005, 23, 2272-2279. [CrossRef]

27. Bennardo, N.; Cheng, A.; Huang, N.; Stark, J.M. Alternative-NHEJ Is a Mechanistically Distinct Pathway of Mammalian Chromosome Break Repair. PLoS Genet. 2008, 4, e1000110. [CrossRef] 